\title{
Bivariate surrogate techniques: Necessity, strengths, and caveats
}

\author{
Ralph G. Andrzejak, ${ }^{1, *}$ Alexander Kraskov, ${ }^{1}$ Harald Stögbauer, ${ }^{1}$ Florian Mormann, ${ }^{2}$ and Thomas Kreuz ${ }^{1,2}$ \\ ${ }^{1}$ John-von-Neumann Institute for Computing, Forschungszentrum Jülich, 52425 Jülich, Germany \\ ${ }^{2}$ Department of Epileptology, University of Bonn, Sigmund-Freud-Straße 25, 53105 Bonn, Germany
}

(Received 28 May 2003; published 15 December 2003)

\begin{abstract}
The concept of surrogates allows testing results from time series analysis against specified null hypotheses. In application to bivariate model dynamics we here compare different types of surrogates, each designed to test against a different null hypothesis, e.g., an underlying bivariate linear stochastic process. Two measures that aim at a characterization of interdependence between nonlinear deterministic dynamics were used as discriminating statistics. We analyze eight different stochastic and deterministic models not only to demonstrate the power of the surrogates, but also to reveal some pitfalls and limitations.

DOI: 10.1103/PhysRevE.68.066202

PACS number(s): 05.45.Tp, 05.45.Xt, 05.10.Ln, 05.40.Ca
\end{abstract}

\section{INTRODUCTION}

Univariate nonlinear time series analysis provides a number of measures for the characterization of nonlinear deterministic dynamics [1]. For most of these measures, however, the ranges of values obtained for nonlinear deterministic dynamics and for linear stochastic dynamics overlap substantially [2]. Therefore, it is often problematic to decide whether a given value of a nonlinear measure reflects an underlying nonlinear deterministic dynamics or whether it is consistent with a linear stochastic model. This ambiguity has been addressed by the method of surrogate data [3], which allows testing results from nonlinear time series analysis against a specified null hypothesis. For this purpose an ensemble of surrogate time series is constructed from the original time series in such a way that they have all properties consistent with this null hypothesis in common with the original, but are otherwise random. A discriminating statistics, which has to be sensitive to at least one property that is inconsistent with the null hypothesis, is calculated for both the original time series and the surrogates. If the result for the original falls outside the range of values obtained for the surrogates, the null hypothesis can be rejected.

In recent years much attention has been paid to the analysis of bivariate and multivariate dynamics. Particular emphasis has been given to physiological and pathological processes such as neuronal dynamics of epilepsy patients [4-10], of healthy volunteers [11-18], or of animal models [19-21]. Further studies investigated cardiodynamics [22] or cardiorespiratory dynamics [23-27].

In these works different algorithms were applied which aim at detection and characterization of interdependence between two nonlinear deterministic dynamical systems ( $X$ and $Y$ ) from the analysis of time series measured simultaneously from the two dynamics [4-6,8,17,19,20,24,26-38]. For the sake of brevity, in this paper we will use the term nonlinear interdependence as a synonym for interdependence between two nonlinear deterministic dynamical systems, notwith-

*Electronic address: r.g.andrzejak@fz-juelich.de standing the often linear character of coupling terms used in model dynamics.

A straightforward approach to characterizing nonlinear interdependence is to quantify the degree to which close states in the state space of $X$ are mapped to close states in the state space of $Y$ and vice versa. This criterion for generalized synchronization is used by a growing number of measures $[4,5,8,17,19,20,27,28,30,38]$, some of which are defined to test for asymmetrical driver-response relationships. As we will demonstrate in this paper, the interpretation of nonlinear bivariate measures can be even more difficult than in the univariate case. Just as for univariate measures, linear autocorrelations of $X$ and $Y$ will affect bivariate nonlinear measures. A further bias can be caused by asymmetries in the statistical properties of $X$ and $Y$. Most importantly, nonlinear bivariate measures may not be able to distinguish between nonlinear interdependence and linear correlations due to additive mixing $[4,8,12,39]$. These very ambiguities motivated the extension of the concept of surrogates to bivariate time series analysis [40-43].

Despite the existence of different algorithms for the generation of bivariate and multivariate surrogate time series, only few studies used them $[5,8,11,12,15-17,19-$ 21,23,25,27]. Most of these studies dealt exclusively with experimental time series of a priori unknown dynamics. The interpretation of a rejection or an acceptance of the null hypotheses of bivariate surrogates, however, remains difficult for unknown dynamics, and investigations of model dynamics are rare exceptions $[8,17,19,23]$. A comprehensive application to model dynamics with well defined properties is still missing and is therefore the major aim of the present study. For this purpose, we have analyzed time series of various stochastic and deterministic model dynamics using two closely related nonlinear bivariate interdependence measures. We discuss the power, but also a number of problems and pitfalls, of different bivariate surrogate techniques.

\section{METHODS}

\section{A. Nonlinear interdependence measures}

Suppose two scalar time series $\left\{x_{n}\right\}$ and $\left\{y_{n}\right\}$ with $n$ $=1, \ldots, N$ have been measured from dynamical systems $X$ 
and $Y$, respectively. At first the dynamics are reconstructed using delay coordinates [44]

$$
\begin{aligned}
& \mathbf{x}_{n}=\left(x_{n}, \ldots, x_{n-(m-1) \tau_{r}}\right), \\
& \mathbf{y}_{n}=\left(y_{n}, \ldots, y_{n-(m-1) \tau_{r}}\right),
\end{aligned}
$$

with an embedding dimension $m$ and a delay time $\tau_{r}$ for $n$ $=1, \ldots, N^{*}$ with $N^{*}=N-(m-1) \tau_{r}$. Let $r_{n, j}$ and $s_{n, j}, j$ $=1, \ldots, k$, denote the time indices of the $k$ nearest Euclidean neighbors of $\mathbf{x}_{n}$ and $\mathbf{y}_{n}$, respectively. Temporally correlated neighbors are excluded by means of a Theiler correction: $\left|r_{n, j}-n\right|>W$ and $\left|s_{n, j}-n\right|>W$ [45]. For each $\mathbf{y}_{n}$, the mean squared Euclidean distance to its $k$ nearest neighbors is given by

$$
R_{n}^{(k)}(Y)=\frac{1}{k} \sum_{j=1}^{k}\left|\mathbf{y}_{n}-\mathbf{y}_{s_{n, j}}\right|^{2},
$$

and the $X$-conditioned mean squared Euclidean distance is defined by replacing $s_{n, j}$ with $r_{n, j}$ :

$$
R_{n}^{(k)}(Y \mid X)=\frac{1}{k} \sum_{j=1}^{k}\left|\mathbf{y}_{n}-\mathbf{y}_{r_{n, j}}\right|^{2} .
$$

The averaged squared distance of $\mathbf{y}_{n}$ to all remaining points in $\left\{\mathbf{y}_{n}\right\}$ is given by

$$
R_{n}(Y)=\frac{1}{N^{*}-1} \sum_{j=1, j \neq n}^{N^{*}}\left|\mathbf{y}_{n}-\mathbf{y}_{j}\right|^{2} .
$$

If the dynamics of $Y$ is independent of $X$, then there is no particular relation between $r_{n, j}$ and $s_{n, j}$, and

$$
R_{n}(Y) \approx R_{n}^{(k)}(Y \mid X) \gg R_{n}^{(k)}(Y)
$$

holds. In contrast, if closeness in $X$ implies closeness in $Y$, then it follows that

$$
R_{n}(Y) \gg R_{n}^{(k)}(Y \mid X) \approx R_{n}^{(k)}(Y) .
$$

Based on these considerations, Arnhold et al. [4] defined the following two measures:

$$
\begin{gathered}
S(Y \mid X)=\frac{1}{N^{*}} \sum_{n=1}^{N^{*}} \frac{R_{n}^{(k)}(Y)}{R_{n}^{(k)}(Y \mid X)}, \\
H(Y \mid X)=\frac{1}{N^{*}} \sum_{n=1}^{N^{*}} \ln \frac{R_{n}(Y)}{R_{n}^{(k)}(Y \mid X)} .
\end{gathered}
$$

If relation (5) holds, it follows that $S(Y \mid X) \rightarrow 0$ and $H(Y \mid X) \rightarrow 0$. Higher values of $S(Y \mid X)$ and $H(Y \mid X)$ are obtained if $Y$ is dependent on $X$, and relation (6) is true. While by construction $0<S(Y \mid X) \leqslant 1$ holds, $H(Y \mid X)$ has no upper bound. Nonetheless, it was found to be more robust against noise and easier to interpret than $S(Y \mid X)$ [39]. That is because the quantity $R_{n}(Y)$ is much less dependent on the structure and dimensionality in $Y$ than $R_{n}^{(k)}(Y)$. To derive a normalized measure from $R_{n}(Y)$, Quian Quiroga et al. [20] therefore defined

$$
N(Y \mid X)=\frac{1}{N^{*}} \sum_{n=1}^{N^{*}} \frac{R_{n}(Y)-R_{n}^{(k)}(Y \mid X)}{R_{n}(Y)} .
$$

However, $N(Y \mid X)=1$ follows only if $R_{n}^{(k)}(Y \mid X)=0$, where $R_{n}^{(k)}(Y \mid X) \geqslant R_{n}^{(k)}(Y) . \quad R_{n}^{(k)}(Y)=0$ in turn holds only for strictly periodic dynamics. In consequence, even in the case of identical synchronization $N(Y \mid X)$ will be smaller than 1 . How much smaller than 1 is mostly determined by $R_{n}^{(k)}(Y)$, a quantity which is strongly influenced by autocorrelations and/or finite dimensionality of $Y$. To minimize this unwanted influence we here suggest modifying the definition of Eq. (9) according to

$$
M^{*}(Y \mid X)=\frac{1}{N^{*}} \sum_{n=1}^{N^{*}} \frac{R_{n}(Y)-R_{n}^{(k)}(Y \mid X)}{R_{n}(Y)-R_{n}^{k}(Y)} .
$$

Since $M^{*}(Y \mid X)$ can have negative values, we define

$$
M(Y \mid X)=\max \left\{M^{*}(Y \mid X), 0\right\} .
$$

The measures $S(X \mid Y)$ and $M(X \mid Y)$ are defined in complete analogy to Eqs. (7) and (11) in order to test whether $X$ is dependent on $Y$. For independent dynamics all four quantities should tend to zero while in the case of identical synchronization all four will reach their maximal value of 1 . In between these two extremes, asymmetries such as $M(Y \mid X)$ $>M(X \mid Y)$ and $S(Y \mid X)>S(X \mid Y)$ can indicate driverresponse relationships. As already discussed in Refs. $[4,8,12,39]$, however, differences in the dynamical properties of $X$ and $Y$ can likewise cause such inequalities. While we will illustrate such an asymmetry by means of one exemplary dynamical system, we will restrict ourselves to results obtained for $S(Y \mid X)$ and $M(Y \mid X)$ for all other dynamics used here.

Rather than optimizing the analysis parameters for each of the different model dynamics, we chose a fixed setting for all dynamics. The calculation of $S$ and $M$ was performed using an embedding dimension of $m=6$, a time delay $\tau=6$ samples. We used a number of $k=5$ nearest neighbors with a Theiler correction of $W=50$ samples.

\section{B. Surrogates}

In this section we will introduce the different types of surrogates used in our study. Each of them has been designed to test against a certain null hypothesis which will be specified in the respective subsections. All null hypotheses share the following common assumptions: The measurement functions by means of which $\left\{x_{n}\right\}$ and $\left\{y_{n}\right\}$ are derived from $X$ and $Y$ are invertible but possibly nonlinear. To preserve such static nonlinearities all surrogate types are based on a permutation of the original sample values. Hence, all surrogates have the amplitude distributions in common with the corresponding original time series. Furthermore, the dynamics under investigation as well as the measurement function are 
assumed to be stationary. Before we describe the different null hypotheses and corresponding types of surrogates, some technical and notational remarks are necessary.

The periodogram estimator of the power spectrum $\left\{\left|s_{k, x}\right|^{2}\right\}=\left\{\mid a_{k, x} e^{\left.i \phi_{k, x}\right|^{2}}\right\}$ of a time series $\left\{x_{n}\right\}$ of length $N$ is defined for $k=-N / 2, \ldots, N / 2$ via the discrete Fourier transform which assumes the time series to represent one period of a periodic signal. For real-valued time series the periodogram is symmetric in its amplitudes and antisymmetric in its phases:

$$
\begin{gathered}
a_{k}=a_{-k}, \\
\phi_{k}=-\phi_{-k} .
\end{gathered}
$$

Furthermore, the zeroth coefficient $s_{0}$ is real-valued and is given by the mean of the time series.

The linear cross correlation function of two real-valued time series $\left\{x_{n}\right\}$ and $\left\{y_{n}\right\}$ of length $N$ is defined by

$$
C_{x y}(\tau)=\left\{\begin{array}{l}
\frac{1}{N-\tau} \sum_{n=0}^{N-\tau-1} x_{n+\tau} y_{n}, \quad \tau \geqslant 0 \\
C_{y x}(-\tau), \quad \tau<0
\end{array}\right.
$$

for $\tau=-(N-1), \ldots, N-1$. For the special case of $\left\{x_{n}\right\}$ $=\left\{y_{n}\right\}$, Eq. (13) defines the linear autocorrelation function $C_{x x}(\tau)$. Note that the number of summands in Eq. (13) decreases for increasing values of the delay so that for $\tau$ $= \pm(N-1)$ only one summand remains. If the time series, however, are assumed to represent each one period of two continuous periodic signals the summation can be continued into the next period:

$$
C_{x y}^{p}(\tau)=\left\{\begin{array}{l}
\frac{1}{N} \sum_{n=0}^{N-1} x_{(n+\tau) \bmod N} y_{n}, \quad \tau \geqslant 0 \\
C_{y x}(-\tau), \quad \tau<0 .
\end{array}\right.
$$

The periodic cross correlation function is connected to the cross spectrum: $\left\{C_{x y}^{p}(\tau)\right\}=\mathcal{F} T\left(\left\{s_{k, x} s_{k, y}^{*}\right\}\right)$, where the asterisk denotes complex conjugation. In consequence, the periodogram and the periodic autocorrelation function are connected via the Wiener Khinchin theorem: $\left\{C_{x x}^{p}(\tau)\right\}$ $=\mathcal{F} T\left(\left\{\left|s_{k, x}\right|^{2}\right\}\right)$.

\section{1. $\mathcal{H}_{0}^{\mathrm{I}}:$ Two independent linear stochastic processes}

To test the null hypothesis that $X$ and $Y$ are two independent linear stochastic processes $\left(\mathcal{H}_{0}^{\mathrm{I}}\right)$, one should preserve the linear autocorrelation of both $\left\{x_{n}\right\}$ and $\left\{y_{n}\right\}$. Any potential nonlinear deterministic structure in $\left\{x_{n}\right\}$ and in $\left\{y_{n}\right\}$ as well as nonlinear interdependence between them should be destroyed by means of a randomization scheme. Furthermore, also a possible significant linear cross correlation needs to be destroyed. The algorithmic expenditure to generate surrogates according to this null hypothesis is comparatively low: Surrogates for both $\left\{x_{n}\right\}$ and $\left\{y_{n}\right\}$ can be generated separately by means of a univariate technique. We here employed a technique that iteratively permutes the original sample values. This scheme is designed to converge to sur- rogates with power spectra practically indistinguishable from the original ones [46]. It is related to a noniterative procedure proposed in Ref. [3] but overcomes a significant problem of the latter technique which was shown to produce surrogates with a systematic bias in the power spectrum $[42,47]$.

As a seed for the iteration scheme, a random shuffle of the original sample values $\left\{x_{n}\right\}^{(0)}$ is generated. (Here, and in the following, we use tilde symbols to denote surrogate time series. No tilde symbols are used, however, if the values of the surrogate are identical to the original values.) Each iteration step $(i)$ consists of the following two subprocedures.

(1) Filtering: calculate the Fourier transformed $\left\{\tilde{a}_{k, x} e^{\left.i \widetilde{\phi}_{k, x}\right\}^{(i)}}\right.$ of $\left\{x_{\tilde{n}}\right\}^{(i)}$ and replace the amplitudes $\left\{\tilde{a}_{k, x}\right\}^{(i)}$ with the original amplitudes $\left\{a_{k, x}\right\}$ but keep the randomized phases $\left\{\widetilde{\phi}_{k, x}\right\}^{(i)}$ fixed. The inverse Fourier transform of $\left\{a_{k, x} e^{\left.i \widetilde{\phi}_{k, x}\right\}^{(i)}}\right.$ results in $\left\{\tilde{x}_{n}\right\}^{(i)}$.

(2) Rescaling: replace the smallest, second smallest, ..., highest value of $\left\{\tilde{x}_{n}\right\}^{(i)}$ with the smallest, second smallest, ... highest value of $\left\{x_{n}\right\}$ resulting in $\left\{x_{\tilde{n}}\right\}$ which is used to start the next iteration step.

Typically, after a few tens or a few hundreds of iteration steps the amplitudes $\left\{\tilde{a}_{k}\right\}^{(i)}$ will have converged to $\left\{a_{k}\right\}$. Equivalently, $C_{\tilde{x x}}^{p}(\tau)^{(i)}$ will have converged to the corresponding original function, and $\left\{x_{\tilde{n}}\right\}^{(i+1)}$ can be used for one surrogate realization [56]. We will refer to surrogates designed to test the null hypothesis of two independent linear stochastic processes as type-I surrogates.

\section{2. $\mathcal{H}_{0}^{\mathrm{II}}$ : Bivariate linear stochastic processes with an arbitrary degree of linear cross correlation}

To test the null hypothesis that $X$ and $Y$ represent a bivariate linear stochastic process with an arbitrary degree of linear cross correlation $\mathcal{H}_{0}^{\text {II }}$ it is necessary to preserve the linear cross correlation between $\left\{x_{n}\right\}$ and $\left\{y_{n}\right\}$ as well as the linear autocorrelation of both time series. As in the preceding section, any potential nonlinear interdependence between $\left\{x_{n}\right\}$ and $\left\{y_{n}\right\}$ or nonlinear deterministic structure in any of the two time series need to be destroyed. In contrast to type-I surrogates, a pair of surrogate time series $\left\{x_{\tilde{n}}\right\}$ and $\left\{y_{\tilde{n}}\right\}$ has to be generated simultaneously by a bivariate algorithm. For the present study we applied a technique proposed by Schreiber and Schmitz [42]. While the generation of the seed for the iteration and the rescaling subprocedure are carried out individually for both $\left\{x_{n}\right\}$ and $\left\{y_{n}\right\}$ according to the preceding section, the filtering subprocedure has to be modified as follows.

For each $k=1, \ldots, N / 2$ the randomized phases $\widetilde{\phi}_{k, x}$ and $\widetilde{\phi}_{k, y}$ are not preserved but replaced with phases $\psi_{k, x}$ and $\psi_{k, y}$, respectively. These are chosen to minimize

$$
h_{k}=\left|e^{i \psi_{k, x}}-e^{\left.i \widetilde{\phi}_{k, x}\right|^{2}}+\right| e^{i \psi_{k, y}}-\left.e^{i \widetilde{\phi}_{k, y}}\right|^{2}
$$

under the constraint

$$
e^{i\left(\psi_{k, x}-\psi_{k, y}\right)}=e^{i\left(\phi_{k, x}-\phi_{k, y}\right)}
$$


where $\phi_{k, x}$ and $\phi_{k, y}$ denote the phases of the original time series. Equation (16) is fulfilled if we choose $\psi_{k, x}$ and $\psi_{k, y}$ such that

$$
\psi_{k, x}-\phi_{k, x}=\psi_{k, y}-\phi_{k, y}=\alpha_{k} .
$$

Under this additional constraint the minimization problem of $h_{k}$ becomes one dimensional, and an appropriate value for $\alpha_{k}$ is found by first eliminating $\psi_{k, x}$ and $\psi_{k, y}$ from Eq. (15) which after some algebra reads

$$
h_{k}=4-2 \cos \left(\alpha_{k}-\widetilde{\phi}_{k, x}+\phi_{k, x}\right)-2 \cos \left(\alpha_{k}-\widetilde{\phi}_{k, y}+\phi_{k, y}\right) \text {. }
$$

This is extremal for

$$
\tan \alpha_{k}=\frac{\sin \left(\widetilde{\phi}_{k, x}-\phi_{k, x}\right)+\sin \left(\widetilde{\phi}_{k, y}-\phi_{k, y}\right)}{\cos \left(\widetilde{\phi}_{k, x}-\phi_{k, x}\right)+\cos \left(\widetilde{\phi}_{k, y}-\phi_{k, y}\right)}
$$

and minimal if $\alpha_{k}$ is taken in the correct quadrant. The phases for negative values of $k$ are chosen according to Eq. (12) to assure the real-valuedness of the back transform. The coefficient for $k=0$ which represents the (zero) mean of the time series can be skipped. In analogy to type-I surrogates, typically a few tens or a few hundreds of iterations are needed for the convergence of $\left\{\tilde{a}_{k, x}\right\}^{(i)}$ and $\left\{\tilde{a}_{k, y}\right\}^{(i)}$ to $\left\{a_{k, x}\right\}$ and $\left\{a_{k, y}\right\}$, respectively. Again, equivalently, $C_{\tilde{x} \tilde{x}}^{p}(\tau)^{(i)}$, $C_{\tilde{y} \tilde{y}}^{p}(\tau)^{(i)}$ converge to the corresponding original values. As proposed in Ref. [40] the cross spectrum and thereby $C_{x y}^{p}(\tau)$ is preserved by construction [Eq. (17)]. We will refer to surrogates designed to test the null hypothesis of a bivariate linear stochastic processes with an arbitrary degree of linear cross correlation as type-II surrogates.

\section{3. $\mathcal{H}_{0}^{\mathrm{III}}$ : Processes with arbitrary structure but without nonlinear interdependence and without significant linear cross correlation}

As an example of a process consistent with $\mathcal{H}_{0}^{\mathrm{III}}$, we assume two independent nonlinear deterministic dynamics which have been measured without any mixing. Because the nonlinear deterministic structure of independent processes is known to be reflected in values of $S$ and $M$, it was proposed to simply use time shifted signals as surrogates, i.e., to calculate synchronization measures in dependence on a relative temporal shift between the two time series [20]. The shifting operation can be carried out quasicontinuously [20] or by choosing a set of random delays [21,27]. By using a periodic boundary condition, i.e., by wrapping around the end of the shifted time series to its beginning, the total length of the time series can be maintained. The range of the possible relative temporal shifts is given by $-N^{*} / 2<\tau_{s}<N^{*} / 2$. The state space trajectory is invariant under the shifting operation with periodic boundary condition, except for those few vectors that include both the last and the first point of the unshifted time series in their components. We will refer to continuously shifted time series with periodic boundary condition as type-IIIa surrogates.
As we shall discuss in Sec. III, however, there is a subtle problem with the use of periodic boundary conditions, and therefore we here introduce type-IIIb surrogates. These are constructed by cutting the middle $N^{*} / 2$ samples of one time series and shifting this half along the other time series without wrapping. The synchronization measure is calculated only for the respective subsegments of $N^{*} / 2$ samples. The range of the relative temporal shift is now given by $-N^{*} / 4$ $<\tau_{s}<N^{*} / 4$. For the calculation of $S(Y \mid X)$ and $M(Y \mid X)$ the middle part of $\left\{y_{n}\right\}$ is shifted along $\left\{x_{n}\right\}$. Here, the middle part of $\left\{y_{n}\right\}$ is always compared to a different sample of $\left\{x_{n}\right\}$. Under stationary conditions, however, the statistical properties of the state space trajectory of the subsegment of $\left\{x_{n}\right\}$ should not change significantly. In consequence, as for type-IIIa the state space structure of both $X$ and $Y$ is preserved for type-IIIb surrogates. Note that numerous alternative ways could be used which always represent a compromise between the maximal shift and the remaining number of samples. In general, values of $S$ and $M$ will depend on the number of data points, and even systematic deviations for smaller number of data points are possible. Such deviations would, however, not affect the validity of type-IIIb surrogates since values are compared only for time series with the same length, here $N^{*} / 2$ samples. Both type-IIIa and type-IIIb surrogates are designed to test the null hypothesis that $X$ and $Y$ can be regarded as processes with arbitrary structure but without nonlinear interdependence and without significant linear cross correlation $\left(\mathcal{H}_{0}^{\mathrm{III}}\right)$. It has to be stressed that neither type-IIIa nor type-IIIb surrogates are surrogates in the classical sense of a Monte Carlo simulation. No distribution of surrogate values of a certain measure is estimated against which the value for the original time series can be compared at a specified level of significance. Rather, rejections can be established if a single peak is found in the dependence of the synchronization measure on the shift $\tau_{s}$.

\section{4. $\mathcal{H}_{0}^{\mathrm{IV}}$ : Processes with arbitrary structure and an arbitrary degree of linear cross correlation but without nonlinear interdependence}

As an example for a process consistent with $\mathcal{H}_{0}^{\mathrm{IV}}$ we assume two independent nonlinear deterministic dynamics which have been measured by some kind of a linear superposition. For such dynamics, $\mathcal{H}_{0}^{\text {III }}$ would be rejected but one could not decide whether this rejection was caused by linear cross correlations or by nonlinear interdependence between $X$ and $Y$. On the other hand, a rejection of $\mathcal{H}_{0}^{\text {II }}$ can be caused by nonlinear interdependence or by nonlinear deterministic structure in $X$ or in $Y$. In consequence, it is not possible to distinguish a linear superposition of independent nonlinear deterministic dynamics from interdependent nonlinear deterministic dynamics by the combination of type-II and typeIIIa, IIIb surrogates because in both cases both null hypotheses would be rejected. This very discrimination, however, appears important and relevant in many applications to dynamical systems in nature, many of which are assumed to exhibit nonlinear deterministic structures $[48,49]$. To test the null hypothesis that $X$ and $Y$ originate from a linear superposition of independent nonlinear deterministic dynamics it 
TABLE I. Overview of the different model systems and their respective parameters. Time series for the two exemplary parameter values given in the fourth and fifth columns are displayed in Fig. 1. Sixth to ninth columns: The letter $C(N)$ indicates that the particular dynamic is (is not) consistent with a certain null hypothesis. As we shall discuss in the text, the consistency of dynamics $H$ with the different null hypotheses depends on the noise level $\zeta$.

\begin{tabular}{|c|c|c|c|c|c|c|c|c|}
\hline & Model & Parameter & Example 1 & Example 2 & $\mathcal{H}_{0}^{\mathrm{I}}$ & $\mathcal{H}_{0}^{\text {II }}$ & $\mathcal{H}_{0}^{\mathrm{III}}$ & $\mathcal{H}_{0}^{\mathrm{IV}}$ \\
\hline$A$ & Independent filtered noise & Cuttoff frequency & $f_{c}=0.04$ & $f_{c}=0.32$ & $C$ & $C$ & $C$ & $C$ \\
\hline$B$ & Bivariate autoregressive process & Coupling strength & $c_{p}=0.28$ & $c_{p}=0.0125$ & $N$ & $C$ & $C$ & $C$ \\
\hline$C$ & Superposition of independent filtered noise & Mixing strength & $q=0.4$ & $q=0$ & $N$ & $C$ & $C$ & $C$ \\
\hline$D$ & Same as $C$, but with nonlinear measurement function & Mixing strength & $q=0.4$ & $q=0$ & $N$ & $C$ & $C$ & $C$ \\
\hline E & Two uncoupled Rössler dynamics & Frequency mismatch & $\nu=0$ & $\nu=-0.00125$ & $N$ & $N$ & $C$ & $C$ \\
\hline$F$ & Linear superposition of two uncoupled Rössler & Mixing strength & $q=0.4$ & $q=0$ & $N$ & $N$ & $N$ & $C$ \\
\hline$G$ & Coupled Rössler-Lorenz dynamics & Coupling strength & $\varepsilon=5$ & $\varepsilon=0$ & $N$ & $N$ & $N$ & $N$ \\
\hline$H$ & Noisy coupled Rössler-Lorenz dynamics & Noise amplitude & $\zeta=4.4$ & $\zeta=0.87$ & $N$ & $C / N$ & $C / N$ & $C / N$ \\
\hline
\end{tabular}

would in principle be necessary to maintain $\left\{x_{n}\right\},\left\{y_{n}\right\}$, and foremost, $C_{x y}^{p}(\tau)$. Obviously, this would leave no degree of freedom for any randomization. In some cases, however, synchronization measures are calculated primarily in one state space. For example, for $S(Y \mid X)$ and $M(Y \mid X)$ all distances are calculated in $Y$ while only indices are used from $X$. In such a case, it might be sufficient to preserve only $\left\{y_{n}\right\}$ and $C_{x y}^{p}(\tau)$ and to randomize $\left\{x_{n}\right\}$. However, at least the linear properties of $\left\{x_{n}\right\}$ characterized by its autocorrelation should be preserved. The constraints to simultaneously preserve $\left\{y_{n}\right\}, C_{x x}^{p}(\tau)$, and $C_{x y}^{p}(\tau)$, however, would still overspecify the problem. The only possible surrogate $\left\{x_{\tilde{n}}\right\}$ would be an exact copy of the original time series $\left\{x_{n}\right\}$. As a way out of this dilemma, Schreiber proposed to preserve the nonperiodic correlation functions $C_{x x}(\tau)$ and $C_{x y}(\tau)$ only up to a certain maximum value $\tau_{\max }$ of the delay. This can be achieved by minimizing a cost function such as

$$
\begin{aligned}
E= & w_{\text {auto }} \sum_{\tau=1}^{\tau_{\max }}\left[C_{x x}(\tau)-C_{\tilde{x x}}(\tau)\right]^{2} \\
& +w_{\text {cross }} \sum_{\tau=-\tau_{\max }}^{\tau_{\max }}\left[C_{x y}(\tau)-C_{x y}(\tau)\right]^{2}
\end{aligned}
$$

by means of simulated annealing [41]. In this context, the cost function is interpreted as the energy of a thermodynamic system which is annealed slowly in order to reach a ground state, i.e., a global minimum of $E$.

Again a random permutation $\left\{x_{\tilde{n}}\right\}^{(0)}$ of the original time series is generated as a seed for the following iterative procedure.

We draw two random indices $i$ and $j$ from $[0, \ldots, N$ $-1]$. We calculate the change of the energy $(\Delta E)$ that would result from the exchange of $x_{i}$ and $x_{j}$. If $\Delta E$ is found to be negative we carry out the exchange, otherwise perform the exchange only with a probability according to a Boltzmann distribution:

$$
p=e^{-\Delta E / T}
$$

After a certain number of tested or performed exchanges, the temperature $T$ of the thermodynamic system is lowered according to

$$
T_{\text {new }}=T_{\text {old }} \alpha
$$

with $1>\alpha \gg 0$. The computation of $\Delta E$ requires only the calculation of those summands of the correlation functions to which $x_{i}$ and $x_{j}$ contribute. Consequently, a calculation of the correlation functions via the Fourier transform would be extremely ineffective. Rather, Eq. (13) can be used, which has the positive side effect that it does not rely on the assumption that the time series represents one period of a continuous signal, which in fact is violated in most realistic situations. Yet the number of iteration steps is typically of the order of several magnitudes higher than that for type-I and type-II surrogates causing very high computational costs even if optimization strategies [41] are employed.

Furthermore, a reasonably fast convergence of $E$ is not guaranteed but can only be reached if the parameters $\alpha$, $w_{\text {auto }}$, and $w_{\text {cross }}$ are adjusted appropriately. In particular, a proper choice of the weights $w_{\text {auto }}$ and $w_{\text {cross }}$ strongly depends on features of the original time series. Therefore, feedback loops are needed to adjust the parameters leading to even higher computational costs. We will refer to surrogates designed to test against the null hypothesis of two processes with arbitrary structure and an arbitrary degree of linear cross correlation but without nonlinear interdependence $\left(\mathcal{H}_{0}^{\mathrm{IV}}\right)$ as type-IV surrogates. For the calculation of $S(Y \mid X)$ and $M(Y \mid X)$ we keep $\left\{y_{n}\right\}$ and randomize $\left\{x_{n}\right\}$.

\section{Data}

We analyzed time series from eight different bivariate dynamics. These models have been chosen to demonstrate the necessity and strengths of bivariate surrogates, but also to reveal caveats related to this approach. All models involved one continuous control parameter, and the dependence on this parameter was studied in detail (cf. Table I). The number of sample points for each pair of time series $\left\{x_{n}\right\}$ and $\left\{y_{n}\right\}$ was $N=4096$. All time series were set to zero mean and unit variance and all formulas were simplified accordingly. Whenever necessary, a sufficient number of preiterations was 

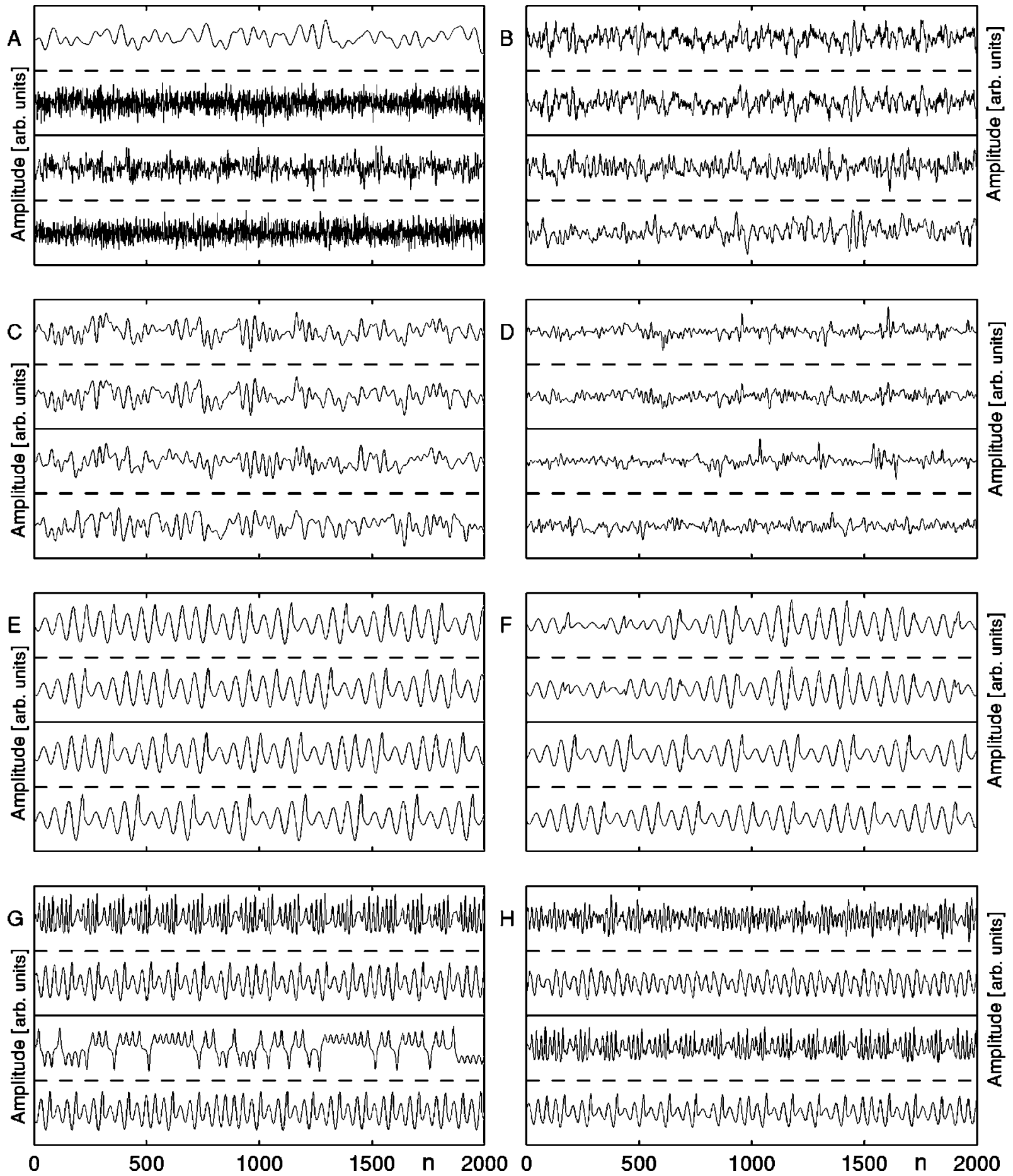

FIG. 1. Segments of exemplary time series of dynamics $A-H$. For each model, two examples are shown. In each panel from top to bottom: $\left\{y_{n}\right\},\left\{x_{n}\right\}$ for example 1 , and $\left\{y_{n}\right\},\left\{x_{n}\right\}$ for example 2 . The parameters of these examples are given in Table I. The quantity $n$ is in units of samples.

carried out for the generation of the models to account for transients. As stated in the preceding section, for the calculation of the discrete Fourier transform time series are implicitly assumed as one period of a continuous signal. Hence, discontinuities in amplitude and slope between the two ends of an otherwise smooth signal would cause spurious frequency components and might lead to a false positive rejection of the null hypotheses of Fourier based surrogates
(type-I and type-II). To circumvent this problem, all time series were cut from longer epochs such that these discontinuities were minimal. For segments of exemplary time series we refer to Fig. 1.

For dynamics $A$, we used independent Gaussian white noise for $\left\{x_{n}\right\}$. For $\left\{y_{n}\right\}$ time series of independent Gaussian white noise were smoothed by means of a sixth-order lowpass butterworth filter. The cutoff frequency $f_{c}$ of the filter 
was decreased stepwise according to $f_{c, p}=0.75^{p}$ with $p$ $=0, \ldots, 14$. Here, the cutoff frequency has been normalized by the bandwidth and hence is dimensionless. Independent realizations were generated for each setting of the filter.

A second-order bivariate autoregressive model was used for the generation of dynamics $B$ :

$$
\left(\begin{array}{c}
x_{n} \\
y_{n}
\end{array}\right)=\sum_{i=1}^{2} \mathbf{A}_{i}\left(\begin{array}{c}
x_{n-i} \\
y_{n-i}
\end{array}\right)+\left(\begin{array}{c}
\xi_{n} \\
\eta_{n}
\end{array}\right)
$$

with

$$
\mathbf{A}_{1}=\left(\begin{array}{cc}
1.85-c_{p} & c_{p} \\
c_{p} & 1.76-c_{p}
\end{array}\right)
$$

and

$$
\mathbf{A}_{2}=\left(\begin{array}{cc}
-0.87 & 0 \\
0 & -0.82
\end{array}\right),
$$

where $\xi_{n}$ and $\eta_{n}$ represent independent identically distributed white noise with zero mean and unit variance. The diffusive coupling strength $c_{p}$ was varied according to $c_{p}$ $=0.0125 \times 1.25^{p}$ with $p=0, \ldots, 19$.

For dynamics $C$ and $D$ two independent realizations of filtered noise, $\left\{v_{n}\right\}$ and $\left\{w_{n}\right\}$, were generated with $f_{c}$ $=0.125$. Subsequently, $\left\{x_{n}\right\}$ and $\left\{y_{n}\right\}$ were derived from a linear superposition of these time series:

$$
\begin{gathered}
x_{n}=(1-q) v_{n}+q w_{n}, \\
y_{n}=g\left[q v_{n}+(1-q) w_{n}\right] .
\end{gathered}
$$

The mixing strength $q$ was increased from $q=0$ (no mixing) to $q=0.5$ (resulting in $\left\{x_{n}\right\}=\left\{y_{n}\right\}$ ) in steps of 0.05 . As measurement functions we used $g(y)=y$ for dynamics $C$ and $g(y)=y \sqrt{|y|}$ for dynamics $D$. Independent realizations of $\left\{v_{n}\right\}$ and $\left\{w_{n}\right\}$ were used for each value of $q$.

Dynamics $E$ and $F$ are based on two nonidentical uncoupled Rössler systems (cf. Refs. [50,51]):

$$
\begin{gathered}
\dot{v}_{1}=-(1+\nu) v_{2}-v_{3}, \\
\dot{v}_{2}=(1+\nu) v_{1}+0.15 v_{2}, \\
\dot{v}_{3}=\left(v_{1}-10\right) v_{3}+0.2, \\
\dot{w}_{1}=-(1-\nu) w_{2}-w_{3}, \\
\dot{w}_{2}=(1-\nu) w_{1}+0.15 w_{2}, \\
\dot{w}_{3}=\left(w_{1}-10\right) w_{3}+0.2 .
\end{gathered}
$$

The quantity $\nu$ which determines the difference in the mean periods of the two Rössler systems was varied from $\nu$ $=-0.0125$ to $\nu=0.0125$ in steps of 0.00125 for dynamics $E$. Time series $\left\{x_{n}\right\}$ and $\left\{y_{n}\right\}$ were sampled at a rate of $d t$ $=0.1$ from $v_{1}$ and $w_{1}$, respectively.
For dynamics $F$, the Rössler dynamics [Eq. (27)] was integrated for a fixed value $\nu=-0.0125$. In analogy to dynamics $C$, the time series $\left\{x_{n}\right\}$ and $\left\{y_{n}\right\}$ were obtained from a linear superposition:

$$
\begin{aligned}
& x_{n}=(1-q) v_{n}+q w_{n}, \\
& y_{n}=q v_{n}+(1-q) w_{n} .
\end{aligned}
$$

Again, the mixing strength $q$ was increased from $q=0$ (no mixing) to $q=0.5$ (resulting in $\left\{x_{n}\right\}=\left\{y_{n}\right\}$ ) in steps of 0.05 .

For dynamics $G$ and $H$ we used a coupled Rössler-Lorenz system:

$$
\begin{gathered}
\dot{v}_{1}=-6\left(v_{2}+v_{3}\right), \\
\dot{v}_{2}=6\left(v_{1}+0.2 v_{2}\right), \\
\dot{v}_{3}=6\left[\left(v_{1}-5.7\right) v_{3}+0.2\right], \\
\dot{w}_{1}=10\left(-w_{1}+w_{2}\right), \\
\dot{w}_{2}=28 w_{1}-w_{2}-w_{1} w_{3}+\varepsilon v_{2}^{2}, \\
\dot{w}_{3}=w_{1} w_{2}-\frac{8}{3} w_{3} .
\end{gathered}
$$

For dynamics $G$, the coupling strength was varied from $\varepsilon$ $=0$ to $\varepsilon=5$ in steps of 0.2 . Time series $\left\{x_{n}\right\}$ and $\left\{y_{n}\right\}$ were sampled directly from $v_{1}$ and $w_{1}$ with $d t=0.025$.

For dynamics $H$, solutions of Eq. (29) for $\varepsilon=5$ were superimposed with isospectral noise at a varying signal to noise ratio ( $\zeta$ measured in units of the variance of the noise-free time series). Independent realizations with randomized initial conditions of the coupled Rössler-Lorenz dynamics were generated for each value of $\zeta$ which was varied according to $\zeta_{p}=0.01 \times 1.5^{p}$ with $p=0, \ldots, 25$.

\section{RESULTS}

Values of the interdependence measures for the different model systems in dependence on the respective parameter readily illustrate the problems related to the interpretation of absolute values of $S$ and $M$ (cf. Fig. 2). The ranges of values overlap substantially. Thus, depending on the setting of the two systems' parameters, the one or the other dynamics appears to be more interdependent. We of course know that for dynamics $G$ the parameter $\varepsilon$ indeed controls the strength of the nonlinear interdependence which is correctly reflected by an increase of $S$ and $M$. However, such an increase can also be caused by static cross correlations due to a linear superposition of univariate linear stochastic processes (dynamics $C$ and $D$ ) or of independent nonlinear deterministic dynamics (dynamics $F$ ). High values of $S$ and $M$ can also be related to dynamical cross correlation of bivariate linear stochastic processes (dynamics B) or can be obtained for two nonlinear deterministic dynamics that are independent but follow similar or identical equations of motions (dynamics $E$ ). In some cases, such as dynamics $A$ or $F$, either only $S$ or only $M$ 

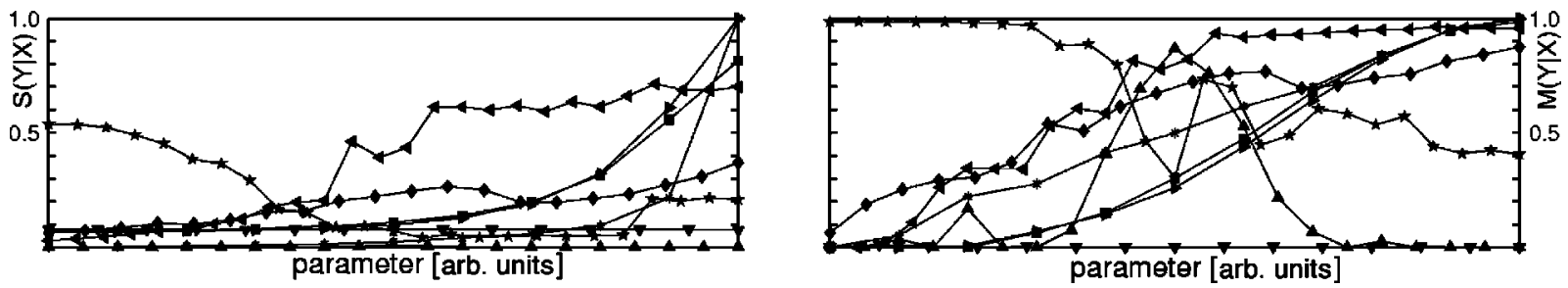

FIG. 2. Values of $S(Y \mid X)$ (left panel) and $M(Y \mid X)$ (right panel) for dynamics $A-H$ in dependence on the respective parameter. $A$, $\nabla$; $B, \diamond ; C, \mathbf{\Delta} ; D, \mathbf{\square} ; E, \mathbf{\Delta} ; F, * ; G, \mathbf{\Delta} ; H, \star$.

exhibits high values while the other measures' values are close to zero. In summary, the interpretation of Fig. 2 appears rather problematic without the use of a priori knowledge about the dynamics, which is of course not what we want for the investigation of unknown dynamics. In consequence, a reliable classification of the different types of dynamics cannot be derived from absolute values of $S$ and $M$ only. To what extent such a classification can be achieved with the help of surrogate time series is the subject of the following discussion. Here, we shall always test first against $\mathcal{H}_{0}^{\mathrm{I}}$ and $\mathcal{H}_{0}^{\mathrm{II}}$. Only if both are rejected, does testing against $\mathcal{H}_{0}^{\mathrm{III}}$ and $\mathcal{H}_{0}^{\mathrm{IV}}$ become necessary. We regard a null hypothesis as rejected also if it is rejected only for $S$ or only for $M$.
For dynamics $A$, values of $S(Y \mid X), S(X \mid Y), M(Y \mid X)$, and $M(X \mid Y)$ are contrasted with the corresponding surrogate distributions in Fig. 3(a). For the complete range of $f_{c}$ both $M(Y \mid X)$ and $M(X \mid Y)$ as well as the corresponding surrogate distributions are very close to zero, correctly reflecting the lack of any interdependence between $X$ and $Y$. In contrast, higher and asymmetric values are established for $S(X \mid Y)$ and $S(Y \mid X)$. This phenomenon, which was also discussed in Ref. [8], can be explained by different autocorrelations of the two independent subsystems. A very narrow range of the surrogates' distribution is found. The narrowness of the distribution of statistical properties of type-I surrogates has been discussed in Refs. [42,47,52] as a potential cause of false
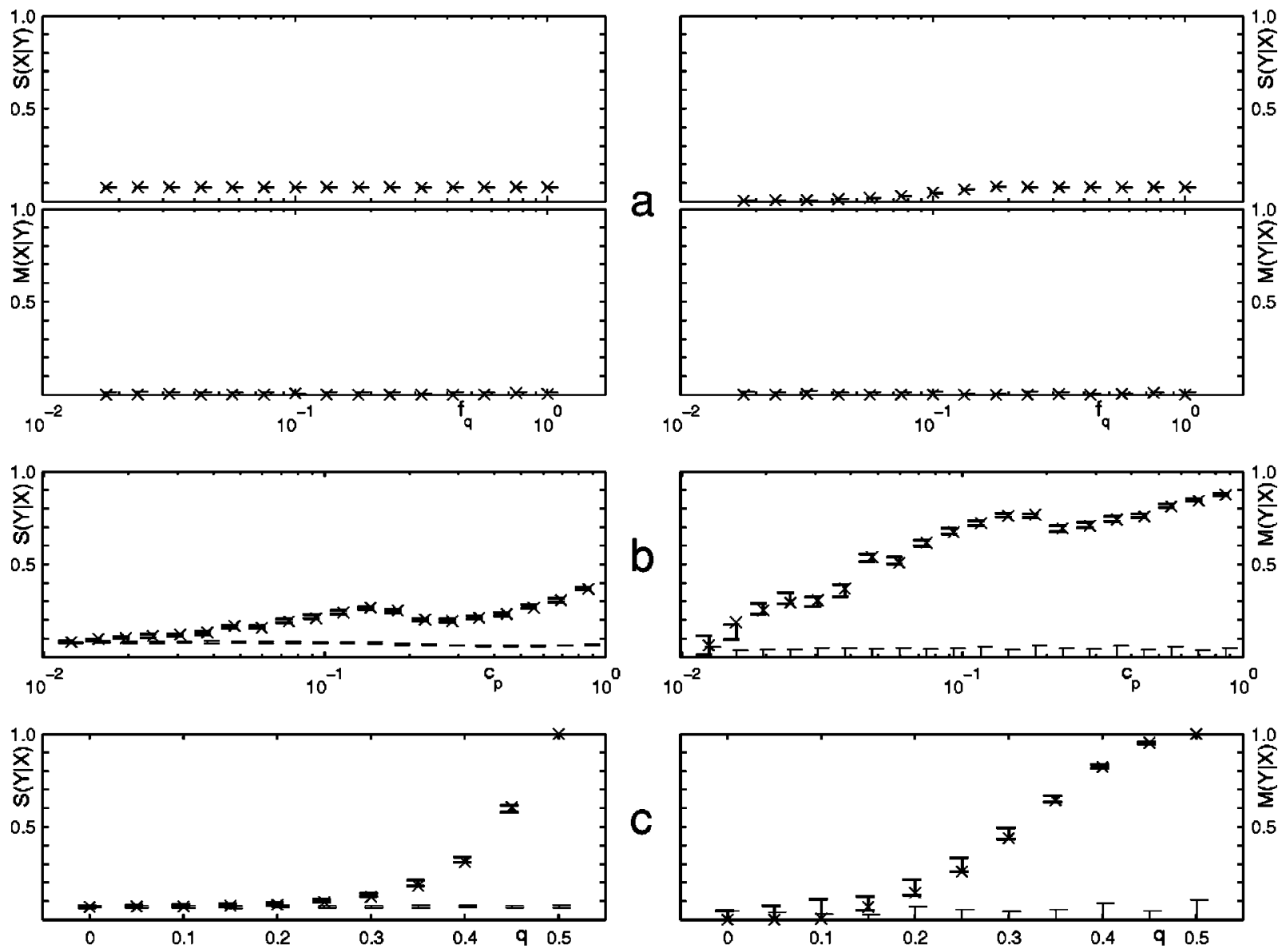

FIG. 3. (a) Values of the nonlinear interdependence measures $S(X \mid Y)$ and $S(Y \mid X)$ (upper panels) and for $M(X \mid Y)$ and $M(Y \mid X)$ (lower panels) for dynamics $A$ vs $f_{q}$. (b) Values of $S(Y \mid X)$ (left panel) and $M(Y \mid X)$ (right panel) for dynamics $B$ in dependence on $c_{p}$. Crosses depict results calculated for the pair of original time series. Normal and bold error bars indicate the range of 39 type-I and type-II surrogates, respectively. (c) Same as (b) for dynamics $C$ vs $q$. 
positive rejections of the null hypothesis. In our case, however, $S$ values are within the surrogate distribution throughout the range of the parameter, and also the asymmetry of $S(X \mid Y)$ and $S(Y \mid X)$ is correctly mimicked by the surrogates. The null hypothesis of two independent univariate linear stochastic processes cannot be rejected for dynamics $A$, which in fact is correct. The decay of $S(Y \mid X)$ for small values of $f_{c}$ can be explained by a decrease of $R_{n}^{(k)}(Y)$ rather than by an increase of $R_{n}^{(k)}(Y \mid X)$ [cf. Eq. (7)]. A fixed number of nearest neighbors in state space is simply found in smaller hypersphere radii for smoother signals, a fact also known to potentially fool estimates of correlation dimension in application to linear stochastic dynamics (cf., e.g., Ref. [2]).

While dynamics $A$ could be used to illustrate how different linear autocorrelations of $X$ and $Y$ can cause asymmetries between $S(Y \mid X)$ and $S(X \mid Y)$ we will restrict ourselves to results for $S(Y \mid X)$ and $M(Y \mid X)$ for dynamics $B-H$. In the following, the distribution of 39 type-II (type-IV) surrogates will be denoted with $\left\{S^{\mathrm{II}}\right\}$ and $\left\{M^{\mathrm{II}}\right\}\left(\left\{S^{\mathrm{IV}}\right\}\right.$ and $\left.\left\{M^{\mathrm{IV}}\right\}\right)$.

For dynamics $B$ the null hypothesis of two independent univariate linear stochastic processes is rejected for higher values of $c_{p}$ [cf. Fig. 3(b)]. This rejection correctly reflects that with increasing values of $c_{p}$ more and more cross correlation between $X$ and $Y$ is introduced. However, at no degree does such a cross correlation contradict the null hypothesis of a bivariate linear stochastic process, which is correctly reflected by the accordance of $S$ and $M$ with $\left\{S^{\mathrm{II}}\right\}$ and $\left\{M^{\mathrm{II}}\right\}$, respectively. The nonmonotonic increase of $S$ and $M$ can be explained by the impact of $c_{p}$ on the spectral properties of $X$ and $Y$ (cf. Fig. 1).

The null hypothesis of two independent univariate linear stochastic processes is also rejected for higher values of $q$ for dynamics $C$ [cf. Fig. 3(c)]. Here the cross correlation between $X$ and $Y$ is introduced via the mixing according to Eq. (26). The accordance of $S$ and $M$ with $\left\{S^{\mathrm{II}}\right\}$ and $\left\{M^{\mathrm{II}}\right\}$ again indicates the correct acceptance of $\mathcal{H}_{0}^{\mathrm{II}}$. As stated above, identical time series are obtained for $q=0.5$ resulting in $S$ $=M=1$. Values of exactly 1 for both $S$ and $M$ are also obtained for all pairs of type-II surrogates for $q=0.5$ because $x_{n}=y_{n} \forall n \Leftrightarrow C_{x y}(\tau=0)=1$ holds. Since type-II surrogates have the linear cross correlation function in common with the original data, pairs of identical surrogate time series are obtained: $x_{\tilde{n}}=y_{\tilde{n}} \forall \tilde{n}$.

The few deviations obtained for dynamics $A-C$ for single values of parameters are consistent with rejections by chance due to a finite value of the nominal size. However, as we shall see in the following, one always has to be aware of the fact that such rejections can also be caused by systematic biases in the surrogates' linear properties. As demonstrated in Fig. 4(a), there is a false rejection of $\mathcal{H}_{0}^{\text {II }}$ for dynamics $D$ throughout the range of $q$. These rejections are caused by a significant deviation of the linear properties of the surrogates, as illustrated in Figs. 4(c,d). Here, the iterative process failed to produce surrogates with linear correlations indistinguishable from those of the original time series. As indicated above it is also possible to stop the algorithm for the generation of type-II surrogates after the filtering step so that the surrogates have the cross correlation function and the peri- odogram exactly in common with the original data. But in that case the deviation in the amplitude distribution caused a false positive rejection of $\mathcal{H}_{0}^{\mathrm{II}}$ (results not shown). Dynamics $D$ are also consistent with $\mathcal{H}_{0}^{\text {IV }}$ but despite the fact that the cross correlation is much better mimicked by type-IV surrogates [cf. Fig. 4(c)], this null hypothesis is rejected for higher values of $q$ [cf. Fig. 4(b)]. These deviations are caused by a mismatch of the autocorrelation or periodogram, respectively [cf. Fig. 4(d)]. This "whitening" of the surrogates' power spectrum could not be reduced sufficiently. Even for extremely low values of the cost function or with the use of additional terms in the cost function, a persistent noisiness of type-IV surrogates could not be suppressed. Whether these problems can be solved by possible generalizations of univariate techniques, which have been designed to include nonmonotonic measurement functions into the null hypothesis [53], shall be the subject of further research. The distinct peaks in the curves of type-IIIb surrogates for dynamics $D$ [cf. Fig. 4(e)] obtained for high values of $q$ indicate the correct rejection of $\mathcal{H}_{0}^{\mathrm{III}}$. We here know that this rejection is caused by the strong linear cross correlation which is introduced by the linear mixing. For unknown dynamics, however, the rejection of $\mathcal{H}_{0}^{\text {III }}$ would not allow deciding whether it is caused by linear cross correlation or by nonlinear interdependence.

A further problem with type-IV surrogates is encountered when we deal with strong cross correlations between the original time series: Type-II and type-IV surrogates are constructed to have the linear cross correlation in common with the original time series. If we interpret a significant linear cross correlation at zero time lag as a similarity relation $(\simeq)$ this reads $\left\{x_{n}\right\} \simeq\left\{y_{n}\right\} \Leftrightarrow\left\{x_{n}\right\} \simeq\left\{y_{n}\right\}$. (The extreme case of $\left\{x_{n}\right\}=\left\{y_{n}\right\} \Leftrightarrow\left\{x_{n}\right\}=\left\{y_{n}\right\}$ has already been discussed along with dynamics $C$ for $q=0.5$.) For type-IV surrogates the additional constraint $\left\{x_{n}\right\}=\left\{x_{n}\right\}$ along with the transitivity of the similarity relation implies $\left\{x_{n}\right\} \simeq\left\{y_{n}\right\} \Rightarrow\left\{y_{n}\right\} \simeq\left\{y_{n}\right\}$. In consequence, when there exists a significant linear cross correlation between $\left\{x_{n}\right\}$ and $\left\{y_{n}\right\}$, type-IV surrogates tend to converge towards a copy of the original time series (cf. Fig. 5). However, even for $q=0.5$ the surrogates do not represent an exact copy of the original time series resulting in $\left\{M^{\mathrm{IV}}\right\}$ $<M$ and $\left\{S^{\mathrm{IV}}\right\}<S$.

Before we proceed to results obtained for deterministic models, let us stress that practically the complete range of definition of both $S$ and $M$ is covered by values obtained for our exemplary stochastic models $A-D$. For the sake of clarity, results of type-I surrogates are not shown for the following dynamics $E-H$. We should mention, however, that $\left\{S^{\mathrm{I}}\right\}$ $\approx\left\{S^{\mathrm{II}}\right\}$ and $\left\{M^{\mathrm{I}}\right\} \approx\left\{M^{\mathrm{II}}\right\}$. That is because a possibly significant cross correlation of type-II surrogates will always increase values of $S$ and $M$.

For dynamics $E$, values of $S$ and $M$ are compared to values of $\left\{S^{\mathrm{II}}\right\}$ and $\left\{M^{\mathrm{II}}\right\}$, respectively, in Fig. 6(a). For a number of $\nu$ values, $M$ significantly deviates from $\left\{M^{\mathrm{II}}\right\}$. Values of $S$ are very close to zero and significantly below $\left\{S^{\mathrm{II}}\right\}$ for small values of $\nu$. We will discuss this seemingly paradoxical finding below and here just note that the null hypothesis of a bivariate linear stochastic process is rejected for dynam- 

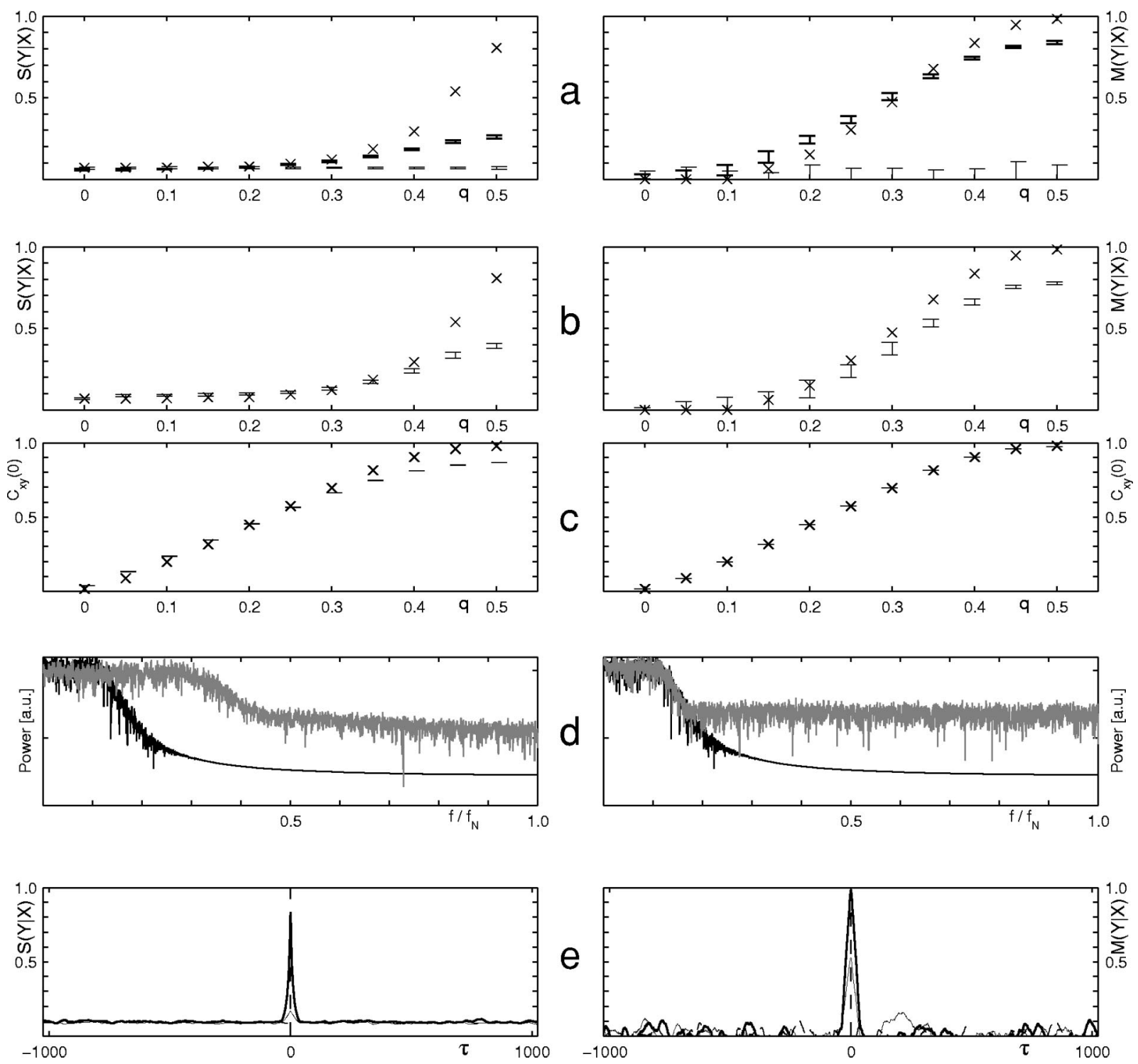

FIG. 4. (a) Values of $S(Y \mid X)$ (left panel) and $M(Y \mid X)$ (right panel) for dynamics $D$ vs $q$. Crosses depict results calculated for the pair of original time series. Normal and bold error bars indicate the range of 39 type-I and type-II surrogates, respectively. (b) Same as (a) but here error bars show the range of 39 type-IV surrogates. (c) Values of the linear cross correlation function at zero time lag. Crosses depict results calculated for the pair of original time series. Error bars in the left and right plot indicate the range of 39 type-II surrogates and type-IV surrogates, respectively. (d) Periodogram of dynamics $D$ for $q=0.3$ (black) in comparison to the periodogram of one exemplary type-II surrogate (left, gray) and one exemplary type-IV surrogate (right, gray). (e) Type-IIIb surrogates for dynamics $D$ : Values of the nonlinear interdependence measures in dependence on the relative time shift $\tau_{s}$ without periodic boundary condition for $q=0.5$ (thick solid line), $q=0.30$ (thin solid line), and $q=0.05$ (dashed line). The quantity $\tau_{s}$ is in units of samples.

ics $E$. Therefore, this hypothesis test does not provide a consistent model for dynamics $E$, necessitating one to test against a different null hypothesis.

Results for type-IIIa surrogates are shown in Fig. 6(b) for three exemplary values of $\nu$. While values of $S$ are very close to zero throughout the range of $\nu$ prominent peaks are found for $M$. Recall that $M\left(\tau_{s}=0\right)$ corresponds to $M(\nu)$ in Fig. 6(a) for a given value of $\nu$. While no clear structure is found for $\nu=-0.0075$, i.e., a $\nu$ value corresponding to the flat tails of $M(\nu)$, broad peaks with maxima at $\tau \approx 0$ are established for $\nu=-0.0025$ and in particular for $\nu=0$, values corresponding to the flanks and maxima of the peaks in $M(\nu)$. So far, an interpretation of these findings for dynam- ics $E$ would need to be as follows: The peakedness of $M(\tau)$ represents a rejection of $\mathcal{H}_{0}^{\mathrm{III}}$, in other words, dynamics $E$ exhibits significant cross correlation and/or nonlinear interdependence. Since $\mathcal{H}_{0}^{\text {II }}$ was also rejected, some kind of nonlinear interdependence might be favored. The small but nonzero time lags of the maxima of $M(\tau)$ could indicate some delay in this interdependence. We know, however, that this interpretation would be wrong, as there is no nonlinear interdependence in dynamics $E$, nor is there any significant linear cross correlation. Hence, this rejection of $\mathcal{H}_{0}^{\mathrm{III}}$ is false.

To disclose the underlying problem it is at first necessary to understand the peaks in the $M(\nu)$ curves. Dynamics $E$ obeys strong periodic components corresponding to long lin- 


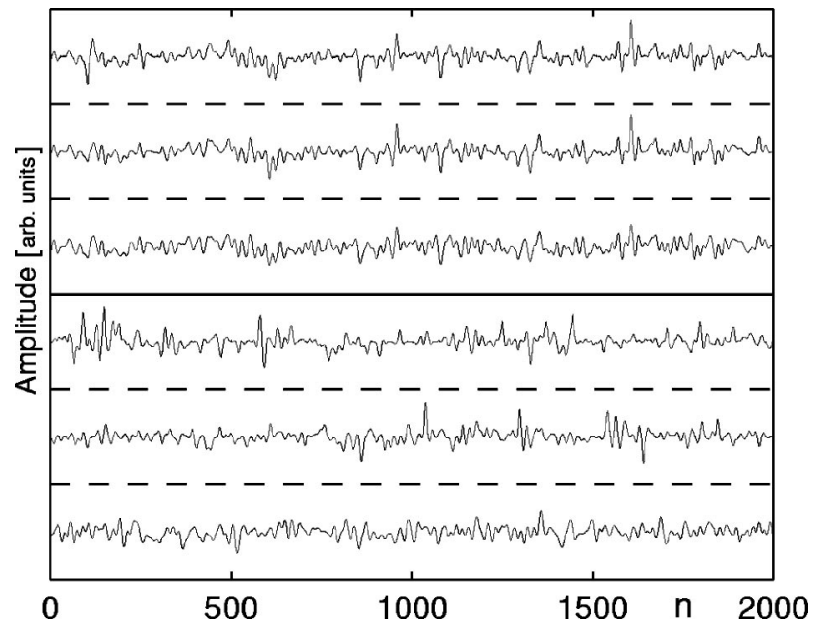

FIG. 5. Segments of exemplary time series of dynamics $C$ and corresponding type-IV surrogates. From bottom to top: $\left\{x_{n}\right\},\left\{y_{n}\right\}$, and $\left\{y_{n}\right\}$ for $q=0.15$ and $\left\{x_{n}\right\},\left\{y_{n}\right\},\left\{y_{n}\right\}$ for $q=0.45$. For the latter value of $q$, which corresponds to a high linear cross correlation, the surrogate $\left\{y_{n}\right\}$ strongly resembles the original time series $\left\{y_{n}\right\}$. The quantity $n$ is in units of samples.

ear autocorrelation times. The mean frequencies of these components for $X$ and $Y$ become similar and identical for small and zero values of $\nu$, respectively. In consequence, both $X$ and $Y$ exhibit almost periodic spatial recurrences with similar recurrence times resulting in high values of $M(\nu$ $\rightarrow 0$ ). Here, the periodic boundary conditions of type-IIIb surrogates will in general introduce a phase slip in the shifted signal which in turn decreases the overall periodicity of recurrences with increasing temporal shifts. These considerations explain the peaks of $M(\tau)$ obtained for dynamics $E$. Doubtless dynamics $E$ is particularly well suited to fully reveal this artifact which might be far less prominent in other cases. But, nonetheless, we here propose to use time shifted surrogates only without periodic boundary condition. Results for these type-IIIb surrogates are shown in Fig. 6(c). No clear peaks are established in the $M(\tau)$ curves: The null hypothesis of two arbitrary but uncorrelated and independent processes is correctly accepted for dynamics $E$.

We still need to discuss why for dynamics $E$ values of $S$ are lower than any of the $\left\{S^{\mathrm{II}}\right\}$ throughout the range of the parameter $\nu$ [cf. Fig. 6(a)]. This can readily be explained by the deterministic structure of the Rössler dynamics which is reflected in low values of $R_{n}^{(k)}(Y)$. The surrogates lack any structure and therefore result in higher values of $R_{n}^{(k)}(Y)$. The same phenomenon is established for dynamics $F$ for which results are shown in Fig. 7(a). Here for $q \leqslant 0.45$ values of $S$ are below the distributions $\left\{S^{\mathrm{II}}\right\}$. Also $M$ deviates significantly from $\left\{M^{\mathrm{I}}\right\}$ for an intermediate range of the mixing parameter $q$. Here, $M$ is higher than any of the $\left\{M^{\mathrm{II}}\right\}$ which might be misinterpreted to indicate nonlinear interdependence for these dynamics. Like for dynamics $C$ the case $q$ $=0.5$ corresponds to identical original time series and pairwise identical type-II surrogate time series. Therefore, all interdependence measures correctly attain their maximal
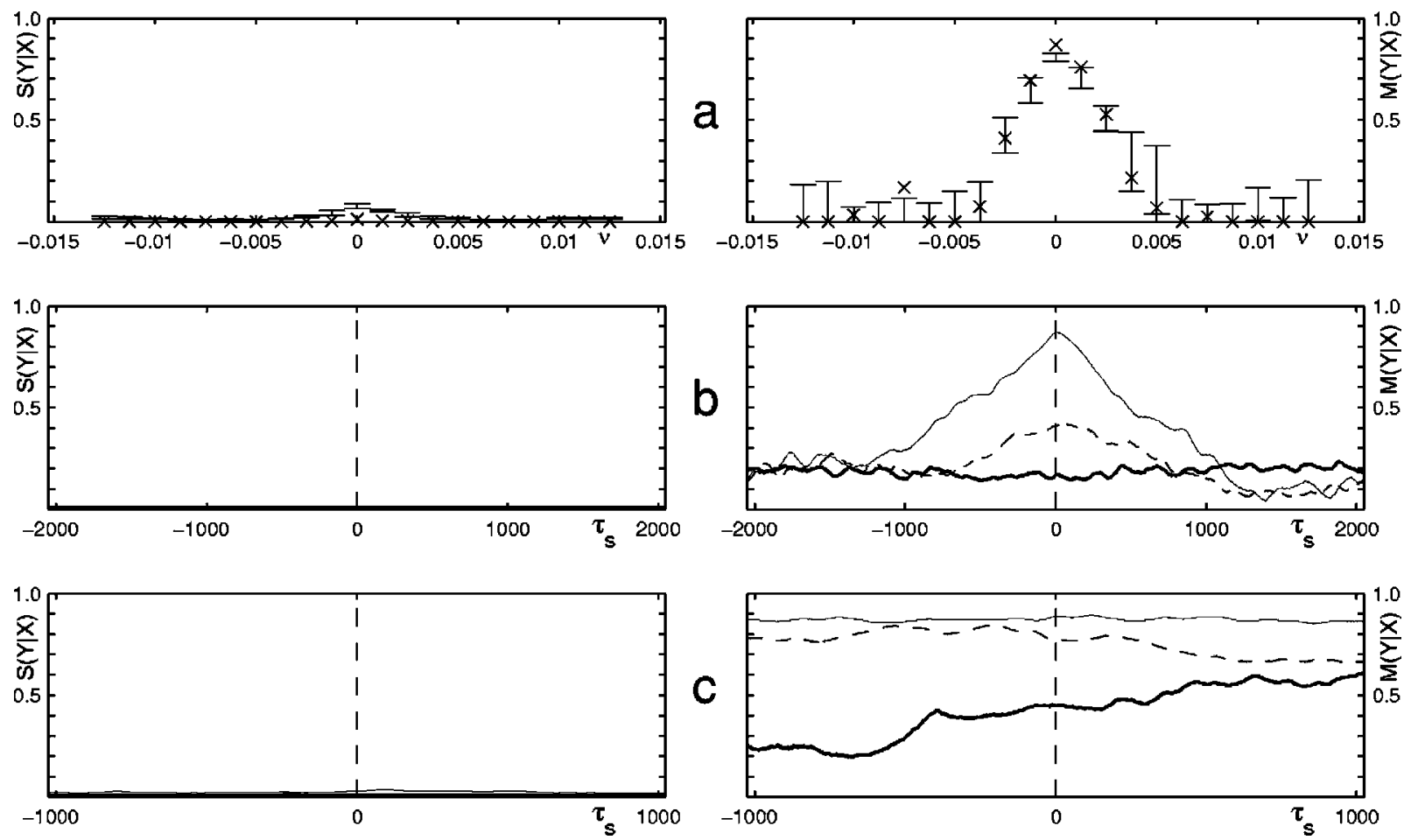

FIG. 6. (a) Values of $S(Y \mid X)$ (left panel) and $M(Y \mid X)$ (right panel) for dynamics $E$ in dependence on $\nu$. Crosses depict results calculated for the pair of original time series. Error bars indicate the range of 39 type-II surrogates. (b) Type-IIIa surrogates for dynamics $E$ : Values of the nonlinear interdependence measures in dependence on the relative time shift $\tau_{s}$ with periodic boundary condition for $\nu=-0.0075$ (thick solid line), $\nu=-0.0025$ (dashed line), and $\nu=0$ (thin solid line). (c) Same as b but without periodic boundary condition (type-IIIb surrogates). For both (c) and (d) $\tau_{s}$ is in units of samples. 

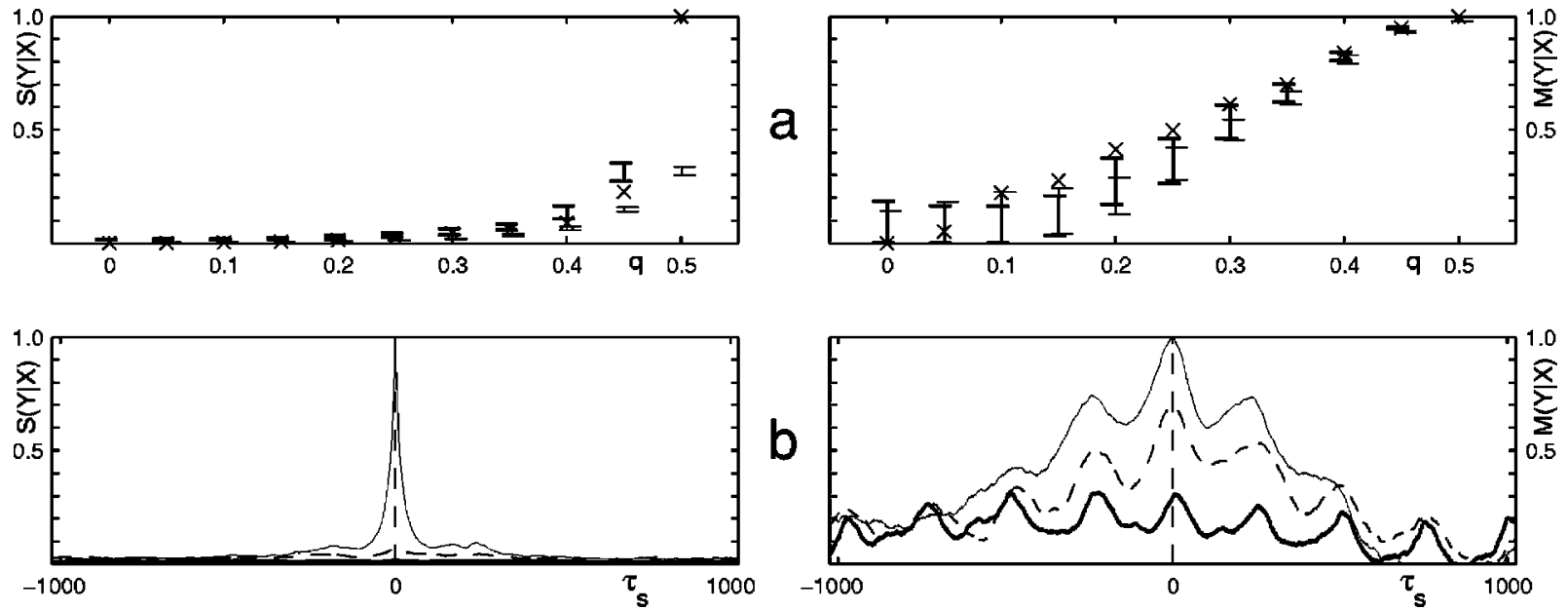

FIG. 7. (a) Values of $S(Y \mid X)$ (left panel) and $M(Y \mid X)$ (right panel) for dynamics $F$ vs $q$. Crosses depict results calculated for the pair of original time series. Bold and normal error bars indicate the range of 39 type-II and type-IV surrogates, respectively. (b) Type-IIIb surrogates for dynamics $F$ : Values of the nonlinear interdependence measures in dependence on the relative shift $\tau_{s}$ without periodic boundary condition for $q=0.2$ (thick solid line), 0.35 (dashed line), and 0.5 (thin solid line). The quantity $\tau_{s}$ is in units of samples.

value of 1 for $q=0.5$. For all other values of $q$, the deterministic structure of the two independent Rössler dynamics causes a rejection of $\mathcal{H}_{0}^{\mathrm{II}}$, necessitating one to test against a different null hypothesis. But also $\mathcal{H}_{0}^{\mathrm{III}}$ is rejected for dynamics $F$ for higher values of $q$ [cf. Fig. 7(b)]. This rejection is correct and can be explained by the cross correlation which is introduced with increasing values of $q$. Despite the consistency of these cross correlations with $\mathcal{H}_{0}^{\text {IV }}$ there are significant deviations of $S$ from $\left\{S^{\mathrm{IV}}\right\}$ and of $M$ from $\left\{M^{\mathrm{IV}}\right\}$, respectively, as can be seen from Fig. 7(a). Only for small values of $q$ do we find an accordance of $S$ with $\left\{S^{\mathrm{IV}}\right\}$. This further supports that the decrease of $S$ below $\left\{S^{\mathrm{II}}\right\}$ is solely related to small values of $R_{n}^{(k)}(Y)$, since these quantities are preserved by construction for type-IV surrogates. But still our results demonstrate a false positive rejection of $\mathcal{H}_{0}^{\mathrm{IV}}$ for dynamics $F$. Therefore, it is not sufficient to preserve the structure of one time series, as is done for the generation of type-IV surrogates, to test against $\mathcal{H}_{0}^{\mathrm{IV}}$.

As a coupled nonlinear deterministic dynamics our example $G$ is not consistent with any of the null hypotheses tested here. Therefore, the rejections of $\mathcal{H}_{0}^{\mathrm{II}}$ and $\mathcal{H}_{0}^{\text {IV }}$ [Fig. $8($ a) $]$ and of $\mathcal{H}_{0}^{\text {III }}$ [Fig. 8(b)] are correct. In analogy to dynamics $F$, values of $S$ are below the distribution $\left\{S^{\mathrm{II}}\right\}$ for low values of the coupling $\varepsilon$. Here, the distributions $\left\{S^{\mathrm{IV}}\right\}$ are closer to the original values and enclose $S$ for $\varepsilon=0$. This finding further demonstrates that for independent dynamics $S$ is mostly dominated by $R_{n}^{(k)}(Y)$, respectively.

While for $\zeta=0$ dynamics $H$ is a nonlinear coupled deterministic dynamics, a purely linear stochastic process is obtained for the limit $\zeta \rightarrow \infty$. Since independent noise is super-
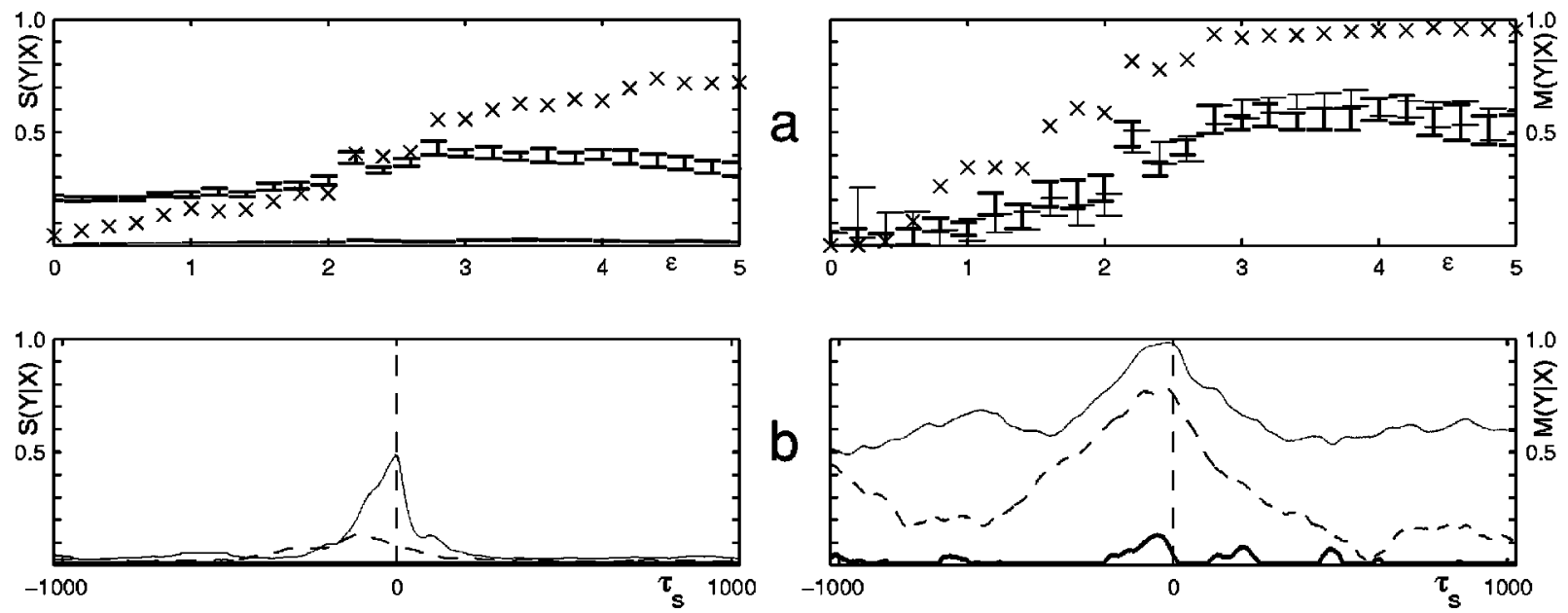

FIG. 8. (a) Values of $S(Y \mid X)$ (left panel) and $M(Y \mid X)$ (right panel) for dynamics $F$ vs $\varepsilon$. Crosses depict results calculated for the pair of original time series. Bold and normal error bars indicate the range of 39 type-II and type-IV surrogates, respectively. (b) Type-IIIb surrogates for dynamics $G$ : Values of the nonlinear interdependence measures in dependence on the relative shift $\tau_{s}$ without periodic boundary condition for $\varepsilon=5$ (thin solid line), 1.8 (dashed line), and 0 (thick solid line). The quantity $\tau_{s}$ is in units of samples. 

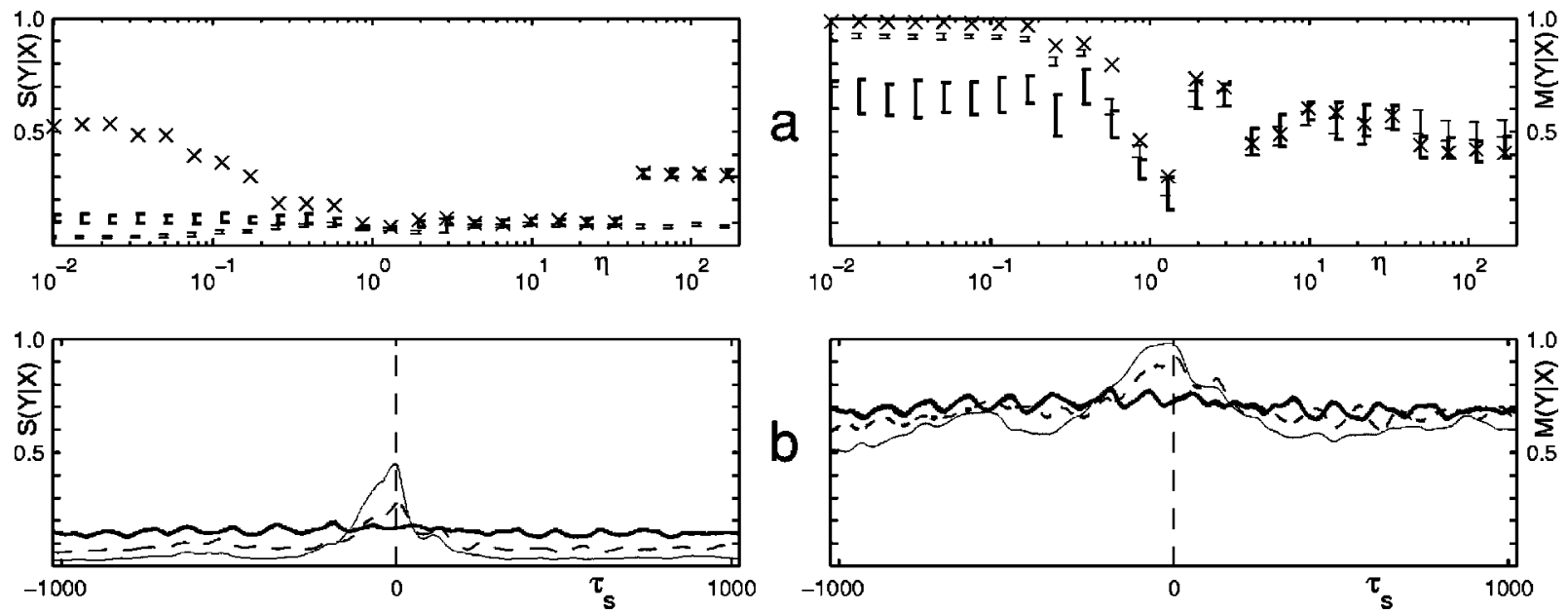

FIG. 9. (a) Values of $S(Y \mid X)$ (left panel) and $M(Y \mid X)$ (right panel) for dynamics $H$ in dependence on $\eta$. Crosses depict results calculated for the pair of original time series. Bold and normal error bars indicate the range of 39 type-II and type-IV surrogates, respectively. b) Type-IIIb surrogates for dynamics $H$ : Values of the nonlinear interdependence measures in dependence on the relative shift $\tau_{s}$ without periodic boundary condition for $\eta=0.05$ (thin solid line), 0.38 (dashed line), and 2.9 (thick solid line). The quantity $\tau_{s}$ is in units of samples.

imposed on $\left\{x_{n}\right\}$ and $\left\{y_{n}\right\}$, no significant linear cross correlation is expected in the purely stochastic extreme. As illustrated in Figs. 9(a,b) all null hypotheses are accepted approximately at a noise level $\eta \approx 1$. In the context of noisy dynamics, surrogates can be used to determine the noise amplitude up to which a certain measure is able to distinguish noisy nonlinear interdependence from isospectral noise with the same linear cross correlation.

\section{DISCUSSION}

Analyzing various bivariate model dynamics, we have demonstrated that the interpretation of measures aiming at a characterization of nonlinear interdependence can be quite problematic. In line with previous studies [4,8,12,39] we showed that different linear and nonlinear properties of the two dynamics which are not necessarily related to nonlinear interdependence strongly influence values of $S$ and $M$. In consequence, these measures do not allow the distinguishing of coupled nonlinear deterministic systems from bivariate linear stochastic processes with different degrees of linear cross correlation. Our results clearly demonstrate that these shortcomings can successfully be approached by the concept of surrogates. A rejection or an acceptance of a surrogate's null hypothesis is nonredundant to the information obtained from the sole use of a nonlinear interdependence measure, and to a great extent a reliable discrimination of different dynamical systems can be derived from a combination of nonlinear interdependence measures with the method of surrogates.

Each of the biases of $S$ and $M$ could be understood using a priori knowledge about the investigated dynamics. However, as stated above, such knowledge is not at hand when we analyze unknown dynamics. Doubtless the understanding of such problems and pitfalls should be used for an improvement of existing measures or the development of new strategies for the characterization of nonlinear interdependence.
But also refined or new concepts will not be infallible and might not account for any known or unforeseen caveats. Hence, the method of surrogates can be regarded as a complementary concept in the progress of nonlinear time series analysis.

However, as also pointed out, e.g., in Refs. [42,47], and demonstrated here by various examples, the method of surrogates should not be misunderstood as an omnipotent and foolproof framework. When surrogates are used to test against a null hypothesis $\mathcal{H}_{0}$ it is very important to keep in mind that the complementary hypothesis, $\mathcal{H}_{1}$, is very comprehensive. Suppose, for example, we had rejected $\mathcal{H}_{0}^{\text {II }}$ with $S$ or $M$ as test statistics. Since these measures were specifically designed to characterize nonlinear interdependence, this rejection might of course correctly indicate an underlying nonlinear interdependence (cf. dynamics $G$ and $H$ ). Nonetheless, a rejection of this null hypothesis at whatever level of significance cannot prove that we are dealing with nonlinear interdependence. Alternative reasons for a rejection can be read from the inversion of the null hypothesis itself: e.g., a non-Gaussian random process, nonstationarity of one or both dynamical systems [54], a nonmonotonic measurement function [47,53], or deterministic structure in the dynamics (cf. dynamics $E$ and $F$ ). Even if one could rule out such reasons, countless further reasons would remain, most of which one might not be aware of. But as demonstrated by dynamics $D$, the surrogate algorithms might also fail to produce time series which really match the original time series in all the specified properties. And as pointed out by Kugiumtzis it is always important to test your surrogates before you test your data [47]. On the other hand, if $\mathcal{H}_{0}^{\text {II }}$ cannot be rejected this does not prove its correctness. The discriminative power of the measure used for the hypothesis test might simply be too low to detect nonlinear interdependence from the analysis of time series with a finite length and limited precision which might furthermore be superimposed with noise (cf. dynamics $H$ ). Strictly speaking, a rejection of 
the null hypothesis of a bivariate linear stochastic process is neither necessary nor sufficient to conclude for the conclusion of a nonlinear interdependence.

Doubtless a number of issues remain open and unsolved. In the context of dynamics $D$ we have investigated many different nonlinear measurement functions and always encountered a significant mismatch of the cross correlation and/or autocorrelation. The most challenging question still is how to discern a linear superposition of independent nonlinear deterministic dynamics from interdependent nonlinear deterministic dynamics.

In closing, we should not overstress these limitations but rather emphasize that the concept of surrogates is a powerful tool in the framework of nonlinear time series analysis. This holds true not only from an academic point of view but also has practical applications: In the context of univariate time series analysis of electroencephalographic recordings of epilepsy patients, we compared the performance of different kinds of measures for the localization of the seizure generating structure [55]. For nonlinear measures, a rather weak performance was obtained which was even surpassed by linear measures. Clearly, the highest performance was obtained for the combination of the nonlinear measures with type-I surrogates. Hence, the surrogates allowed one to extract additional information which could not be derived by nonlinear measures alone. These findings appear to be of high value for diagnostic purposes. To what extent such promising findings can be extrapolated to the case of bivariate nonlinear measures in application to electroencephalographic recordings of epilepsy patients is subject to current investigations.

\section{ACKNOWLEDGMENTS}

We are grateful to Peter Grassberger, Klaus Lehnertz, and Christoph Rieke for countless valuable discussions. We wish to thank Peter Grassberger for carefully reading this manuscript. T.K. and F.M. acknowledge support from the Deutsche Forschungsgemeinschaft.
[1] H. Kantz and T. Schreiber, Nonlinear Time Series Analysis (Cambridge University Press, Cambridge, U.K., 1997).

[2] A.R. Osborne and A. Provenzale, Physica D 35, 357 (1989).

[3] J. Theiler, S. Eubank, A. Longtin, B. Galdrikian, and J.D. Farmer, Physica D 58, 77 (1992).

[4] J. Arnhold, K. Lehnertz, P. Grassberger, and C.E. Elger, Physica D 134, 419 (1999).

[5] M. Le Van Quyen, J. Martinerie, C. Adam, and F. Varela, Physica D 127, 250 (1999).

[6] F. Mormann, K. Lehnertz, P. David, and C.E. Elger, Physica D 144, 358 (2000).

[7] J. Fell, P. Klaver, K. Lehnertz, T. Grunwald, C. Schaller, C. Elger, and G. Fernández, Nat. Neurosci. 4, 1259 (2001).

[8] C.J. Stam and B.W. van Dijk, Physica D 163, 236 (2002).

[9] F. Mormann, T. Kreuz, R.G. Andrzejak, P. David, K. Lehnertz, and C.E. Elger, Epilepsy Res. 53, 173 (2003).

[10] F. Mormann, R.G. Andrzejak, T. Kreuz, C. Rieke, P. David, C.E. Elger, and K. Lehnertz, Phys. Rev. E 67, 021912 (2003).

[11] S.A.R.B. Rombouts, R.W.M. Keunen, and C.J. Stam, Phys. Lett. A 202, 352 (1995).

[12] E. Pereda, R. Rial, A. Gamundi, and J. González, Physica D 148, 147 (2001).

[13] J. Bhattacharya, H. Petsche, and E. Pereda, J. Neurosci. 21, 6329 (2001).

[14] J. Bhattacharya and H. Petsche, Phys. Rev. E 64, 012902 (2001).

[15] M. Breakspear and J.R. Terry, Neuroimage 16, 822 (2002).

[16] E. Pereda, S. Mañas, L. De Vera, J.M. Garrido, S. López, and J. González, Neurosci. Lett. 337, 101 (2003).

[17] J.R. Terry and M. Breakspear, Biol. Cybern. 88, 129 (2003).

[18] P.A. Tass, T. Fieseler, J. Dammers, K. Dolan, P. Morosan, M. Majtanik, F. Boers, A. Muren, K. Zilles, and G.R. Fink, Phys. Rev. Lett. 90, 088101 (2003).

[19] S.J. Schiff, P. So, T. Chang, R.E. Burke, and T. Sauer, Phys. Rev. E 54, 6708 (1996).
[20] R. Quian Quiroga, A. Kraskov, T. Kreuz, and P. Grassberger, Phys. Rev. E 65, 041903 (2002).

[21] T.I. Netoff and S.J. Schiff, J. Neurosci. 22, 7297 (2002).

[22] F. Censi, V. Barbaro, P. Bartolini, G. Galcagnini, A. Michelucci, and S. Cerutti, Biol. Cybern. 85, 195 (2001).

[23] M. Paluš, Phys. Lett. A 213, 138 (1996).

[24] M. Paluš, Phys. Lett. A 235, 341 (1997).

[25] M. Paluš and D. Hoyer, IEEE Eng. Med. Biol. Mag. 17, 40 (1998).

[26] M.G. Rosenblum, L. Cimponeriu, A. Bezerianos, A. Patzak, and R. Mrowka, Phys. Rev. E 65, 041909 (2002).

[27] J. Bhattacharya, E. Pereda, and H. Petsche, IEEE Trans. Syst. Man Cybern. B 33, 85 (2003).

[28] A. Cenys, G. Lasiene, and K. Pyragas, Physica D 52, 332 (1991).

[29] H. Kantz, Phys. Rev. E 49, 5091 (1994).

[30] N.F. Rulkov, M.M. Sushchik, L.S. Tsimring, and H.D.I. Abarbanel, Phys. Rev. E 51, 980 (1995).

[31] P. Tass, M.G. Rosenblum, J. Weule, J. Kurths, A. Pikovsky, J. Volkmann, A. Schnitzler, and H.J. Freund, Phys. Rev. Lett. 81, 3291 (1998).

[32] P.J. Franaszczuk and G.K. Bergey, Biol. Cybern. 81, 3 (1999).

[33] J.P. Lachaux, E. Rodriguez, J. Martinerie, and F.J. Varela, Hum. Brain Mapp 8, 194 (1999).

[34] T. Schreiber, Phys. Rev. Lett. 85, 461 (2000).

[35] M.G. Rosenblum and A.S. Pikovsky, Phys. Rev. E 64, 045202(R) (2001).

[36] M. Wiesenfeldt, U. Parlitz, and W. Lauterborn, Int. J. Bifurcation Chaos Appl. Sci. Eng. 11, 2217 (2001).

[37] A. Pikovsky, M. Rosenblum, and J. Kurths, Synchronization. A Universal Concept in Nonlinear Sciences (Cambridge University Press, Cambridge, U.K., 2001).

[38] L. Kocarev and U. Parlitz, Phys. Rev. Lett. 76, 1816 (1996).

[39] R. Quian Quiroga, J. Arnhold, and P. Grassberger, Phys. Rev. E 61, 5142 (2000). 
[40] D. Prichard and J. Theiler, Phys. Rev. Lett. 73, 951 (1994).

[41] T. Schreiber, Phys. Rev. Lett. 80, 2105 (1998).

[42] T. Schreiber and A. Schmitz, Physica D 142, 346 (2000).

[43] K.T. Dolan and A. Neiman, Phys. Rev. E 65, 026108 (2002).

[44] F. Takens, in Dynamical Systems and Turbulence, edited by D.A. Rand and L.-S. Young, Lecture Notes in Mathematics, Vol. 898 (Springer-Verlag, Berlin, 1980), pp. 366-381.

[45] J. Theiler, Phys. Rev. A 34, 2427 (1986).

[46] T. Schreiber and A. Schmitz, Phys. Rev. Lett. 77, 635 (1996).

[47] D. Kugiumtzis, Phys. Rev. E 60, 2808 (1999).

[48] R.G. Andrzejak, G. Widman, K. Lehnertz, P. David, and C.E. Elger, Epilepsy Res. 44, 129 (2001).

[49] R.G. Andrzejak, K. Lehnertz, F. Mormann, C. Rieke, P. David, and C.E. Elger, Phys. Rev. E 64, 061907 (2001).

[50] M.G. Rosenblum, A.S. Pikovsky, and J. Kurths, Phys. Rev. Lett. 76, 1804 (1996)

[51] O.E. Rössler, Phys. Lett. A 57, 397 (1976).

[52] K.T. Dolan and M.L. Spano, Phys. Rev. E 64, 046128 (2001).

[53] D. Kugiumtzis, Phys. Rev. E 62, R25 (2000).

[54] J. Timmer, Phys. Rev. E 58, 5153 (1998).

[55] R.G. Andrzejak (unpublished).

[56] Alternatively, $\left\{\tilde{x}_{n}\right\}^{(i)}$ could be used as a surrogate. In that case the power spectrum would be identical to the original, and the amplitude distribution would be practically indistinguishable from that of the original time series (cf. Ref. [47]). 